\title{
An Individual-Based Model of Influenza in Nosocomial Environments
}

\author{
Boon Som Ong ${ }^{1}$, Mark Chen ${ }^{2}$, Vernon Lee ${ }^{2}$, and Joc Cing Tay ${ }^{1, *}$ \\ ${ }^{1}$ ROSS Scientific Pte Ltd \\ Innovation Centre, Units 211-212, 16 Nanyang Drive \\ Singapore 637722 \\ "joccingaross-scientific.com \\ ${ }^{2}$ Department of Clinical Epidemiology, Tan Tock Seng Hospital \\ Moulmein Road, Singapore 30843
}

\begin{abstract}
Traditional approaches in epidemiological modeling assume a fully mixed population with uniform contact rates. These assumptions are inaccurate in a real epidemic. We propose an agent-based and spatially explicit epidemiological model to simulate the spread of influenza for nosocomial environments with high heterogeneity in interactions and susceptibilities. A field survey was conducted to obtain the activity patterns of individuals in a ward of Tan Tock Seng Hospital in Singapore. The data collected supports modeling of social behaviors constrained by roles and physical locations so as to achieve a highly precise simulation of the ward's activity. Our results validate the long-standing belief that within the ward, influenza is typically transmitted through staff and less directly between patients, thereby emphasizing the importance of stafforiented prophylaxis. The model predicts that outbreak size (and attack rate) will increase exponentially with increasing disease infectiousness beyond a certain threshold but eventually tapers due to a target-limited finite population. The latter constraint also gives rise to a peak in epidemic duration (at the threshold level of infectiousness) that decreases to a steady value for increasing infectiousness. Finally, the results show that the rate of increase in distinct cumulated contacts will be highest within the first 24 hours and gives the highest yield for contact tracing among patients that had prolonged periods of nonisolation. We conclude that agent-based models are a necessary and viable tool for validating epidemiological beliefs and for prediction of disease dynamics when local environmental and host factors are sufficiently heterogeneous.
\end{abstract}

Keywords: Agent-based modeling, Spatially-explicit model, Epidemiology, Influenza, Contact patterns.

\section{Introduction}

During the SARS crisis, hospitals were found to be especially vulnerable to outbreaks [1-3]. Hospitals are also susceptible to nosocomial influenza, and rapid crossinfection between healthcare workers and patients can occur [4-6]. In spite of this, there has been little work in simulating the potential spread of infections in the hospital setting, with a granularity that allows policy makers and infection control 
practitioners to explore the utility and potential impact of various hospital outbreaks and infection control measures. We have therefore chosen, 1) to base the geographic and spatial context of our epidemiological model in which the disease outbreak is based on a hospital environment and, 2) to use agents whose behaviors are based on surveyed activity data of patients and healthcare workers.

We have designed and developed a spatially explicit agent-based epidemiological simulation model called ASINE (which stands for Agent-based Simulator for Infections in the Nosocomial Environment). This model can simulate the dynamics of disease spread through person-to-person contact among the staff and patient population for a particular ward at Tan Tock Seng Hospital (TTSH) in Singapore. While hospital infections have traditionally been modeled using compartmental models, our use of a spatially explicit agent-based simulation is driven by the fact that, in a hospital environment: 1) Individuals interact with each other locally, 2) Individuals are mobile but may be restricted to certain areas, and 3) The individual environment is heterogeneous [7, 8]. Although individual-based models have been used to model the spread of community influenza [7-10], but such an approach, to our knowledge, has not been applied to nosocomial influenza.

\section{The Spread of Influenza within a Hospital Ward}

As alluded to in our introduction, the primary motivation for our work arose out of the experience of nosocomial SARS outbreaks in 2003, and the threat which pandemic influenza may pose to the hospital environment. From this section onwards, we will refer primarily to influenza, as an example of an infectious disease which can potentially be spread in the hospital through staff and patient interactions.

In a typical hospital environment, the main venues for human traffic are within the clinical wards; these were also the key locations where outbreaks were observed during the SARS epidemic in Singapore [1]. Influenza is predominantly spread from person to person, by droplet spray or by direct or indirect (e.g. via fomites) contact with nasopharyngeal secretions [6]. In our model, the geographic context is the spatial environment of $\mathrm{CDC}^{1}$ ward 71.

\subsection{Modeling the Environment}

We implemented a Geographic Information System (GIS) as a data model for a twodimensional schematic map that represents the environment of interest, in which individuals performs their social activities $[11,12]$. The spatial environment only consists of location objects in specific positions with no explicit path information. Therefore, a graph is used to provide the navigational structure for agents to move within the ward $[13,14]$. Each location can be thought of a node in the graph and thus an edge can then be added between two nodes to denote that a path exists between them.

For each location object, we specify a Cartesian coordinate for its position and a rectangle of certain width and height for its shape (say for a bed) in the two dimensional environment. The topology for the CDC ward was thereby approximated in this manner in accordance to our onsite inspection of the ward.

${ }^{1}$ Communicable Disease Centre, TTSH. 


\subsection{Modeling the Human Population at CDC Ward 71}

The healthcare staff can be categorized as: doctors, nurses and health attendants. There is only one clerk and one cleaner. There are many types of patients, but we have categorized them into two types: ambulant and non-ambulant. Ambulant patients are allowed to move around the ward area but for a non-ambulant patient, he/she would be bedridden for the whole duration of stay at the ward. The last type of agent is a visitor, who may visit the patients. In summary, the population at CDC ward 71 (which we modeled) comprises:

- 18 nurses, 6 for each shift.

- 3 health attendants, 1 for each shift.

- 4 doctors, who do their rounds at the ward daily.

- 1 ward clerk. There is only 1 shift for the ward clerk.

- 1 ward cleaner. There is only 1 shift for the ward cleaner.

- Number of patients (ambulant and non-ambulant) and visitors can be parameterized during initialization.

\subsection{Modeling Agent Behaviors}

There are two types of routines - standard and miscellaneous. Healthcare workers have standard routines to follow during a work shift. Nurses need to carry out tasks like taking parameters for patients and bed turnings for non-ambulant patients. Health attendants need to serve meals during meal timings and doctors usually make their rounds in the morning. These standard routines occur during certain times of the day and they must be carried out. Apart from these standard routines, different types of individuals each have a set of activities that may be performed. These activities are categorized as miscellaneous routines an agent performs. For instance, a ward clerk may only visit administrative areas like the doctor's office or the nursing station. A visitor may only visit the patient's room and nursing station, but is out of bounds to the staff room. By definition, the visitor may also choose not to visit a patient. Hence each patient can have 0 or more visitors during the visitation hour. Each individual agent performs such activities or actions probabilistically. The algorithm for the selection of an action is based on roulette wheel selection where each action is associated with a probability value that corresponds to its fitness. The fitter the action, the greater the chance the agent will perform this action.

The social interactions of each individual are simulated on a daily basis with activity patterns obtained from a field survey. This field survey helps to derive the sets of routines mentioned previously that an agent has to carry out. The survey method was sample-based and purely observational. Movements of representative healthcare staff, patients and visitors was observed during an average work day, so as to establish the frequency, duration and intensity of contacts between healthcare staff, patients and their visitors. The ethics review committee of the National Healthcare Group, Singapore, was consulted, with approval obtained, to ensure that the conduct of the field survey respected privacy and ethical consults.

For each location $x$ visited by an individual $y$ (upon observation of $y$ during the survey), the probability of visiting $x$ is calculated based on the observed frequencies of visit to $x$ given by $N_{x}$ divided by the total frequencies of visit to all locations by $y$, 
given by $N$. For example, a total of 50 activities for a particular nurse were observed (during the survey). Out of these 50 activities, 10 of them are activities performed at the nursing station. So the probability of a nurse going to a nursing station is $1 / 5$. The actual type of activities performed by each individual is not relevant. The duration an agent $a_{i}$ spends at location $x$ is drawn from a normal distribution based on the frequencies of visit and the amount of time associated with each visit.

\subsection{Modeling Disease Transmission}

Epidemics are usually described using a set of states; namely, susceptible (S), infected (E), infectious (I) and recovered/removed (R) [15]. Depending on the disease's natural history, an epidemiological model can be described using the SEIR, SEIS, SIR, or SIS pattern as shown in Fig. 1. Fig. 1 illustrates a finite state machine (FSM) which describes the possible state transitions of an infectious disease. The possible set of state transitions that can be obtained from the FSM is SEIR, SEIS, SIR and SIS. The FSM allows us to model diseases of different natural history through alternative state transition routes.

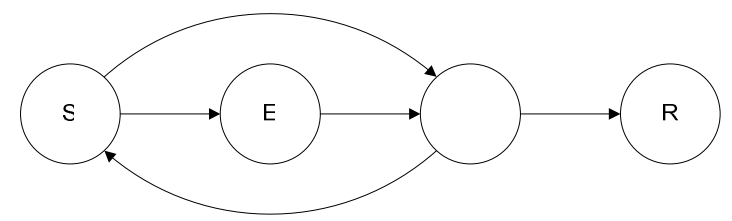

Fig. 1. Possible state transitions of an infectious disease

To simulate the pathogenesis of a disease within a host and transmission between hosts, each individual is associated with a disease model that describes the health state of that individual (using discrete states, susceptible, infected, infectious and recovered). A disease is modeled as an agent and is responsible for transiting between epidemic states and performs computations for infecting other susceptible human agents. The joint preconditions for successful influenza transmission between two agents are 1) both agents must first collocate at a location in order for influenza to spread, and 2) distance between infector agent $x$ and infectee agent $y$ must be within a certain radius. In a spacious room therefore, the disease may not be transmitted so easily. In our model, the infection radius is defined as twice the size of the human agent.

Transmission probability, $\beta$, is defined as the transmissibility of the infectious agent multiplied by the susceptibility of the susceptible agent, which is based on unittime per contact with the following formula:

$$
\text { Unit-time per contact transmission probability, } \beta^{1}=1-(1-\beta)^{T / T^{1}}
$$
where $T=$ Newtonian time and $T^{1}=$ simulation time.

Both transmissibility and susceptibility depend on the instantaneous health state of the individual. The latter is a random variable drawn from a normal distribution with a mean of 0.5 and standard deviation of 0.5 . The range is between 0 (indicates severely 
immune-suppressed) and 1 [8]. Therefore, a healthy person may be less susceptible to infection and less likely to transmit the disease.

All human agents are initialized to be at a susceptible state. The infected and infectious states are both associated with a non-zero time period. When an infectious agent infects a susceptible agent, the susceptible agent will move to the infected state. After the infected period has expired, the agent will move to the infectious state. This is the state where the disease becomes contagious and transmission to other agents can occur at this state. After the infectious period has expired, the agent recovers. Mortality is currently not modeled.

\section{Model Validation and Results}

We designed several experiments with two aims in mind. The first experiment $(A)$ aimed to validate our agent-based epidemiological model in terms of its ability to simulate the ward environment. The second and third experiments ( $B$ and $C$ ) are samples of the type of results we can obtain from this model, which may be useful in guiding control measures.

A) Contact patterns between individuals within the ward environment

Table 1. Contact patterns between individuals within the ward environment

\begin{tabular}{|c|c|c|c|c|c|c|c|c|c|c|}
\hline & \multicolumn{9}{|c|}{ Distinct contacts of index for 1 day, by individual type } \\
\hline & & Cleaner & Clerk & Doctor & $\begin{array}{l}\text { Health } \\
\text { Attendant }\end{array}$ & Nurse & $\begin{array}{l}\text { Ambulant } \\
\text { Patient }\end{array}$ & \begin{tabular}{|l|} 
Non- \\
ambulant \\
Patient
\end{tabular} & Visitor & $\begin{array}{l}\text { Total } \\
\text { Contacts }\end{array}$ \\
\hline \multirow{8}{*}{ 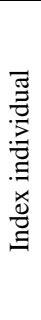 } & Cleaner & 0 & 0.94 & 0.04 & 1.76 & 10.12 & 0.4 & 0.15 & 0.72 & 14.13 \\
\hline & Clerk & 0.89 & 0 & 1.72 & 1.85 & 14.28 & 1.21 & 0 & 3.96 & 23.91 \\
\hline & Doctor & 0 & 0.37 & 2.83 & 0.2 & 5.62 & 7.85 & 14.65 & 4.35 & 35.87 \\
\hline & $\begin{array}{l}\text { Health } \\
\text { Attendant }\end{array}$ & 0.64 & 0.67 & 0.21 & 0 & 8.21 & 1.27 & 0.3 & 2.06 & 13.36 \\
\hline & Nurse & 0.6 & 0.85 & 1.19 & 1.36 & 12.89 & 4.06 & 5.65 & 3.54 & 30.14 \\
\hline & $\begin{array}{l}\text { Ambulant } \\
\text { Patient } \\
\end{array}$ & 0.05 & 0.16 & 3.45 & 0.4 & 8.18 & 2.36 & 0 & 1.91 & 16.51 \\
\hline & $\begin{array}{l}\text { Non-ambulant } \\
\text { Patient }\end{array}$ & 0.01 & 0 & 3.58 & 0.03 & 7.03 & 0 & 0 & 1.29 & 11.94 \\
\hline & Visitor & 0.04 & \begin{tabular}{|l|}
0.09 \\
\end{tabular} & 0.4 & 0.19 & 1.86 & 0.57 & 0.57 & 2.46 & 6.18 \\
\hline
\end{tabular}

Table 1 shows the average number of distinct contacts (from 100 realizations) encountered by a putative index individual within the course of a 24-hour period. We see that the contact patterns in the ward which are generated by the model resemble what we expect of a ward environment. For example, the clerk has a high number of contacts mainly with the nursing staff, due to her central location at the nursing station; she has however, minimal contact with patients. The doctors, on the other hand, have a high number of contacts with nurses, patients and visitors; they also have the highest number of contacts amongst all staff types. When we look at patients, we see that patients have less contacts overall, and that patients have few contacts with other patients; in particular, we see that non-ambulant patients are unlikely to have contact with other patients. The model thus affirms the current opinions that, in many nosocomial diseases, transmission is not occurring from direct patient-to-patient contact but through healthcare workers as vectors. 


\section{B) Outbreak size and path-length for an infectious disease with different transmis- sion probabilities}

We can also use our model to simulate other diseases which may, in the future, cause outbreaks spread by direct person-to-person contact. The set of parameters which have been used to describe the biology and natural history of influenza is shown in Table 2. In this set of experiments, we simulated the propagation of the entire outbreak (i.e. all generations of cases) and then calculated the simulated outbreak size, the total attack rate (including staff, patients and visitors), the epidemic duration, and the number of generations of cases.

Table 2. Disease parameters for influenza (adopted from [10])

\begin{tabular}{|l|l|l|}
\cline { 2 - 3 } \multicolumn{1}{c|}{} & \multicolumn{2}{c|}{ Duration (in days) } \\
\cline { 2 - 3 } \multicolumn{1}{c|}{} & Infected state & Infectious state \\
\hline Minimum & 1 & 3 \\
\hline Maximum & 3 & 6 \\
\hline Mean & 1.9 & 4.1 \\
\hline
\end{tabular}

Again, the parameter describing the infectiousness of the disease is unknown, so we simulated a range of values for infectiousness $(\beta)$, as shown in Fig. 2a. We see that, below a certain threshold value of infectiousness, the average outbreak size is very small; this is because, at these values, the index case on the average produces less than one other infectious case $\left(R_{0}<1\right)$, therefore no propagated transmission is possible. With higher levels of infectiousness, the outbreak size has a near linear association (on a logarithmic scale) with infectiousness. Further increase in infectiousness, however, only has a marginal effect on outbreak size since the ward environment is a finite population and almost all individuals who can be infected would have been infected; this is illustrated by Fig. $2 b$, which shows the attack rates approaching $100 \%$ at values of infectiousness exceeding 0.1 .

When looking at epidemic duration and the maximum number of generations within an outbreak, an interesting pattern emerges. At lower levels of infectiousness, epidemic duration increases with increasing infectiousness (Fig. 2c). This is because of the likelihood that the epidemic will generate successive generations of cases (Fig. 2d). However, with higher levels of infectiousness, epidemic durations decrease; this is because, when the average number of cases infected by an infectious patient increases, the finite number of individuals within the ward environment can be infected in fewer generations than at lower levels of infectiousness.

C) Outcome and yield of contact tracing for simulated outbreaks with different infection parameters

Fig. 3a and Fig. 3b simulate the dynamics of a commonly used intervention, that of contact tracing; the situation simulated is one where the ward environment is exposed to an infectious index case for a number of days before the case is identified and isolated. For Fig. 3a, we explore the number of distinct contacts that would be generated over the infectious period if the index were a patient, or any of the staff types shown in the picture. We see that staff have far more contacts than patients, and nonambulant patients have the least number of contacts. The number of distinct contacts 
(a)

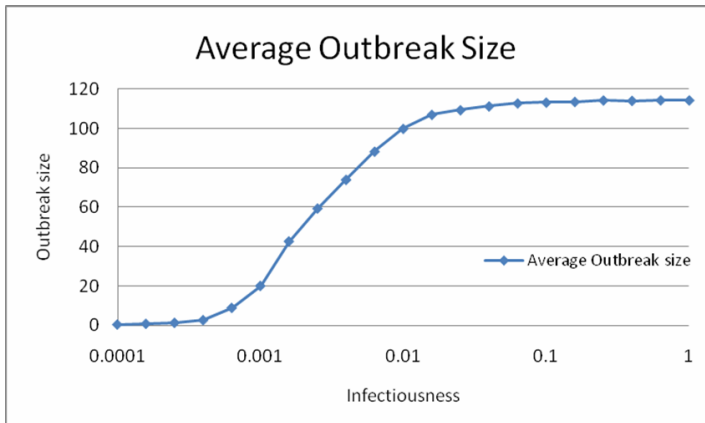

(b)

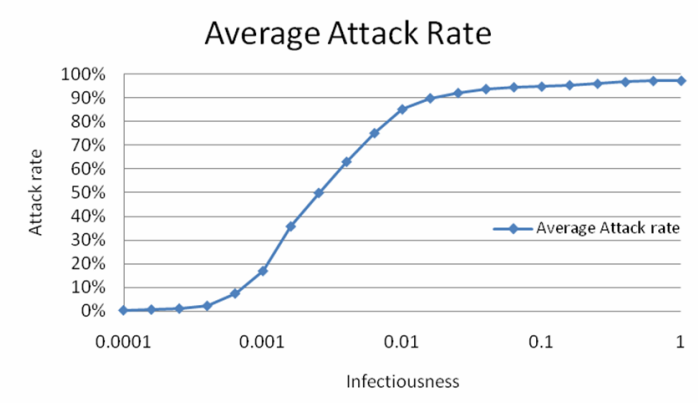

(c)

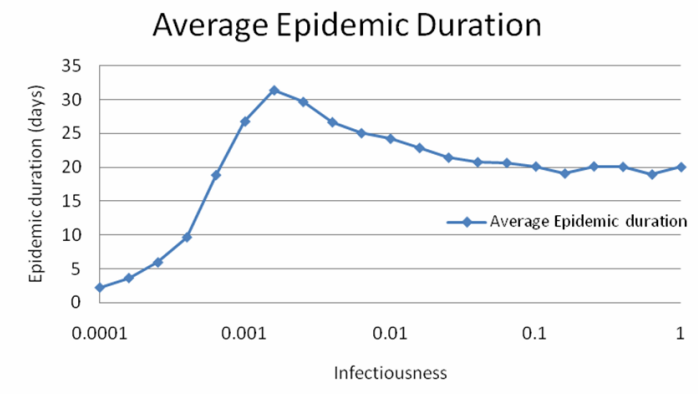

(d)

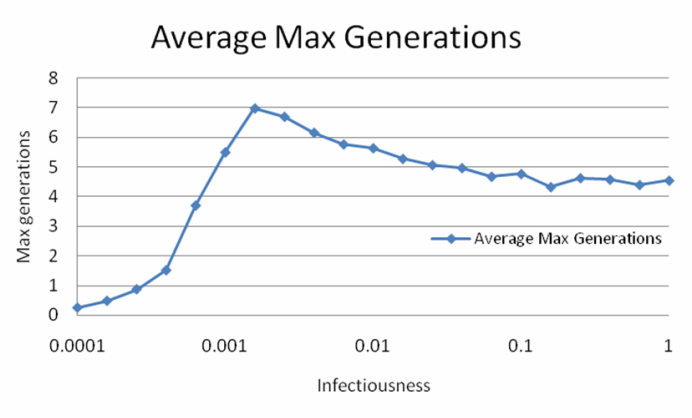

Fig. 2. Outbreak size and path length of influenza 
(a)

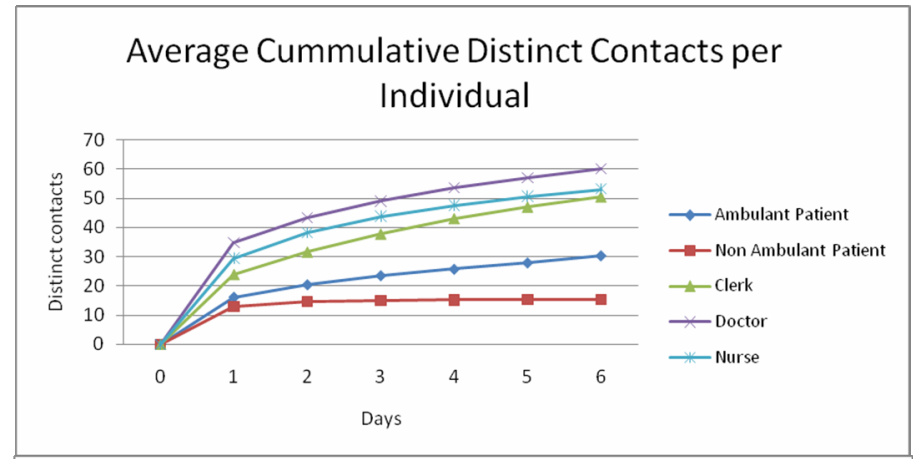

(b)

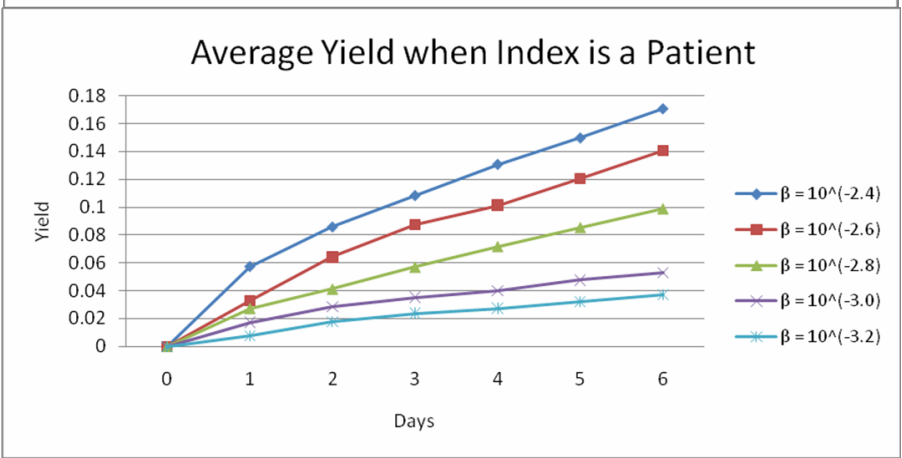

Fig. 3. Outcome and yield of contact tracing

accumulated over time is interesting to note; the sharpest increase is between time zero and day 1; this is because, within the first 24 hours, an index case (in particular in the case of patients), would have met most of the individuals which he/she will ever meet within the ward environment. The result of this cumulative contact pattern translates into the patterns observed in Fig. $3 \mathrm{~b}$ when we look at the yield of contact tracing, when the index case is a patient. If uniform infectiousness is assumed over time, then the cumulative contacts infected by an index case increases with the days that the patient is left without isolation at a faster rate than the increase in the number of contacts, with the result being that the yield of contact tracing is higher for cases who have not been isolated for a longer period, regardless of the level of infectiousness assumed.

\section{Conclusions}

We have designed and developed an individual-based epidemiological simulation model that can accurately simulate the spread of influenza within a ward at the Communicable Disease Centre of Tan Tock Seng Hospital in Singapore. As influenza is typically spread by droplet or direct person-to-person contact, the basic interactivity and social structure of the domain would be of paramount importance. As such, we undertook field surveys of the movement patterns of staff, patients and their visitors. These movements result from visitation patterns, bed and nurse-patient allocation 
methods, and from miscellaneous activities such as visitations to various staff rooms, pantries, washrooms, or taking work breaks and mandatory activities like bed turning, taking of parameters, administration of medication and shift change. We also employed a two dimensional topology for the physical structure of the ward to constrain the navigational movement of individuals. Disease transmission was based on an individualized SEIR model without morbidity and mortality. Heterogeneity in individual health statuses and interaction patterns determine local transmissibility and form the measurable dynamics of disease spread for parameters such as epidemic duration and attack rate. The resulting individual-based, stochastic model of influenza spread within a constrained environment with finite population was validated with epidemiologists through a number of experiments. We established the long-standing belief that within the wards, influenza is typically transmitted through staff and less directly between patients, thereby emphasizing the importance of staff-oriented prophylaxis. Also, results show that outbreak size (and attack rate) increases exponentially with increasing disease infectiousness beyond a certain threshold but tapers eventually due to a target-limited finite population constraint. The latter constraint also gave rise to a peak in epidemic duration (at the threshold level of infectiousness) that decreases to a steady value for increasing infectiousness. Finally, we showed that the rate of increase in distinct cumulated contacts was highest within the first 24 hours and gave the highest yield for contact tracing among patients that had longer periods of non-isolation. Through this project, we showed that agent-based models are a necessary tool for validating epidemiological beliefs and for prediction of disease dynamics when local environmental and host factors are sufficiently heterogeneous.

Acknowledgments. We would like to thank CDC, Tan Tock Seng Hospital of Singapore for providing needed data clearance and access to the ward and its staff. In particular, A/Prof. Leo Yee Sin, Dr. Angela Chow, Staff Nurse Quek Lee Kheng and student helpers Ms. Guo Zaiyi and Ms. Christine Ong.

\section{References}

1. Heng, B.H., Lim, S.W.: Epidemiology and control of SARS in Singapore. Epidemiological News Bulletin, Ministry of Health Singapore 29, 42-47 (2003)

2. Skowronski, D.M., Astell, C., Brunham, R.C., Low, D.E., Petric, M., Roper, R.L., Talbot, P.J., Tam, T., Babiuk, L.: Severe acute respiratory syndrome (SARS): a year in review. Annual Review of Medicine 56, 357-381 (2005)

3. Yu, I.T.S., Sung, J.J.Y.: The epidemiology of the outbreak of severe acute respiratory syndrome (SARS) in Hong Kong-what we do know and what we don't. Epidemiology and Infection 132, 781-786 (2004)

4. Salgado, C.D., Farr, B.M., Hall, K.K., Hayden, F.G.: Influenza in the acute hospital setting. Lancet Infectious Diseases 2, 145-155 (2002)

5. Sartor, C., Zandotti, C., Romain, F., Jacomo, V., Simon, S., Atlan-Gepner, C., Sambuc, R., Vialettes, B., Drancourt, M.: Disruption of services in an internal medicine unit due to a nosocomial influenza outbreak. Infection control and hospital epidemiology 23, 615-619 (2002)

6. Stott, D.J., Kerr, G., Carman, W.F.: Nosocomial transmission of influenza. Occupational Medicine 52, 249-253 (2002) 
7. Bian, L.: A conceptual framework for an individual-based spatially explicit epidemiological model. Environment and Planning B: Planning and Design 31, 381-395 (2004)

8. Dunham, J.B.: An Agent-Based Spatially Explicit Epidemiological Model in MASON. Journal of Artificial Societies and Social Simulation 9 (2005)

9. Ferguson, N.M., Cummings, D.A., Fraser, C., Cajka, J.C., Cooley, P.C., Burke, D.S.: Strategies for mitigating an influenza pandemic. Nature 442, 448-452 (2006)

10. Longini Jr., I.M., Halloran, M.E., Nizam, A., Yang, Y.: Containing Pandemic Influenza with Antiviral Agents. American Journal of Epidemiology 159, 623-633 (2004)

11. Crooks, A.T.: Exploring Cities using Agent-based Models and GIS. In: Proceedings of the Agent 2006 Conference on Social Agents: Results and Prospects, University of Chicago and Argonne National Laboratory, Chicago, IL (2006),

http: / /www.agent2006.anl.gov/2006procpdf/Crooks_Agent_2006. pdf

12. Gonçavels, A.S., Rodrigues, A., Correia, L.: Multi-Agent Simulation Within Geographic Information Systems. In: Coelho, H., Espinasse, B. (eds.) Proceedings of 5th Workshop on Agent-Based Simulation (2004)

13. Buckland, M.: Programming game AI by example. Wordware Pub. (2005)

14. Smed, J., Hakonen, H.: Algorithms and Networking for Computer Games. Wiley, Chichester (2006)

15. Hethcote, H.W.: The Mathematics of Infectious Diseases. SIAM Review 42, 599-653 (2000) 